\title{
METRIZABILITY OF COMPACT CONVEX SETS
}

\author{
BY \\ H. H. CORSON
}

\begin{abstract}
It is proved that a compact convex set is metrizable if the set of extreme points is the continuous image of a complete separable metric space.
\end{abstract}

0. Introduction. Let $E$ be the set of extreme points of a compact convex set $K$ in a locally convex vector space. If $K$ is metrizable, it is well known that $E$ is completely metrizable. This paper presents the measure theory necessary to prove this theorem in $\S 5$. If $E$ is the continuous image of a complete separable metric space, then $K$ is metrizable. Our objective is to present this theorem, and to make the material on capacities and maximal measures more understandable to someone with an average background in general topology.

Of course, the measure theory is not developed from scratch. However, the necessary background has been reduced to four theorems (Theorems 2, 3, 4 and 8) which are stated just as they are used here. For the reader's convenience we refer for the proofs to just one book, that by Paul A. Meyer [1], but it is certain that the reader will have seen each theorem before, at least in some special form.

It will be observed that a more general theorem is proved than that stated above. The property of the set of extreme points $E$ that is used is that $E$ is $K$-analytic, as defined in $\S 1$. This generalization has the properties it should have: The theorems and their proofs seem simpler and more understandable in this context. In fact, it has turned out that any proof which first occurred to me and which used special properties of complete separable metric spaces could be simplified by changing the context. Also, this class of spaces is very large since each compact space, and each complete separable metric space (hence each locally compact space with a countable base) is $K$-analytic.

This note is arranged as follows:

\$1. Contains definitions and a theorem stating the equivalence of various definitions of $K$-analytic. This theorem is not used; it is presented for the reader who is familiar with one but not all of the definitions. Therefore, the reader should begin with $\S 2$.

\$2. Deals with outer measures and the more general concept of capacities.

§3. Presents some results on extending a measure and related theorems.

$\$ 4$. Includes results on the topology of a collection of measures.

Received by the editors July 1, 1969.

AMS 1969 Subject Classifications. Primary 2810, 5435, 5240.

Key Words and Phrases. Convex set, metrizable, extreme points, analytic sets.

Copyright (C) 1970, American Mathematical Society 
§5. Presents some special properties of measures on compact convex sets, and the theorem referred to above is proved.

Most of these results are due, at least in their present form, to G. Choquet; and I am indebted to him for pointing out to me how Theorem 2 could be applied here.

1. Definitions. We say that a set $E$ is topologized by a collection $\mathcal{O}$ of real functions iff $E$ has the weakest topology such that each $f \in \mathcal{O}$ is continuous. By a topological space $E$ we will always mean that $E$ is topologized by some such $\mathcal{O}$, that is, $E$ is completely regular.

(a) For any topological space $E, \mathscr{K}(E)$ will denote the collection of compact subsets of $E$.

(b) $\mathscr{B}(E)$ will denote the $\sigma$-algebra generated by $\mathscr{K}(E)$.

(c) $\mathscr{F}(E)$ will denote the $\sigma$-algebra generated by the closed subsets of $E$.

(d) If $\mu$ is a measure on the $\sigma$-algebra $\mathscr{E}$ and $\mathscr{N}$ is the collection of all subsets of elements of $\mathscr{E}$ which have measure zero, then $\mathscr{M}_{\mu}$ will denote the $\sigma$-algebra generated by $\mathscr{E} \cup \mathscr{N}$.

Consider these four properties which can apply to a subset $A$ of $E$.

(e) There is an upper semicontinuous map $f$ of $N^{N}$ into $K(E)$ such that $A=\bigcup_{s \in N^{N}} f(s)$.

(f) There is a space $F$ and $B \subset E \times F, B \in(\mathscr{K}(E) \times \mathscr{K}(F))_{\sigma \delta}$ such that $\pi_{1}(B)=A$.

(g) $A$ is the continuous image of some $B \in(\mathscr{K}(F))_{\sigma \delta}$ for some space $F$.

(h) $A$ is a subset of a compact $E$ and $A$ can be derived from Souslin's operation $A$.

THEOREM 1. (e) iff (f) iff (g) iff (h).

Proof. See [2] and [3].

(i) If $A$ satisfies one of these conditions then $A$ is said to be $K$-analytic. (Note that this property is preserved under taking closed subsets, or countable unions or intersections, or taking continuous images.)

2. Capacities. We will be concerned with measures on $K$-analytic spaces. These are best investigated through the outer measures associated with them. The main result is a consequence of a general result concerning capacities.

Definition. $I$ is a capacity on $E$, where $E$ is a topological space and $I: 2^{E} \rightarrow[0, \infty]$ iff

(i) $A \supset B \Rightarrow I(A) \geqq I(B)$,

(ii) $A_{1} \subset A_{2} \subset \cdots, A_{i} \subset E$, $\Rightarrow I\left(\bigcup A_{i}\right)=\sup I\left(A_{i}\right)$,

(iii) $A_{1} \supset A_{2} \supset \cdots, A_{i} \in \mathscr{K}(E), \Rightarrow I\left(\bigcap A_{i}\right)=\inf I\left(A_{i}\right)$

DefinItion. $A \subset E$ is capacitable (for a capacity $I$ ) iff $I(A)=\sup _{B \in \mathscr{K}(E) ; B \subset A} I(B)$.

THEOREM 2. Let I be a capacity on $E$. Then every $K$-analytic set $A$ in $E$ is capacitable. 
Proof. See [1, p. 39].

The connection between these results and measures is given by the next theorem. Let $\mathscr{A}$ denote a $\sigma$-algebra on $E$ containing $\mathscr{K}(E)$.

THEOREM 3. Let $\mu$ be a nonnegative measure on $\mathscr{A}$. Let $\mu^{*}(A)=\inf _{B \in \mathscr{A} ; B \supset A} \mu(B)$. Then $\mu^{*}$ (the outer measure) is a capacity which is an extension of $\mu$.

Proof. [1, p. 42].

3. Measures. $(F, \mathscr{F}) \stackrel{f}{\rightarrow}(E, \mathscr{E})$. Let $E$ and $F$ be sets with $\sigma$-algebras $\mathscr{E}$ and $\mathscr{F}$ respectively. If $f$ is a measurable function from $F$ into $E$ and $\mu$ is a measure on $\mathscr{F}$, let $\tilde{f}(\mu)$ be the measure on $\mathscr{E}$ defined by $\tilde{f}(\mu)(A)=\mu\left(f^{-1}(A)\right)$. We will be concerned with the questions: Which measures on $\mathscr{E}$ are of the form $\tilde{f}(\mu)$, and is the measure $\mu$ uniquely determined by its image?

Warning. Henceforth we restrict our attention to nonnegative, finite measures. By well-known results it is often possible to reduce a problem concerning more general measures to this case. However, this will not be used.

If $F=E, f$ is the identity $i$, and $\mathscr{F}=\mathscr{M}_{\mu}$ as in Definition (d), we have this answer to the above problem:

THEOREM 4. Each $\mu$ on $\mathscr{E}$ can be uniquely extended to a measure $\tilde{\mu}$ on $\mathscr{M}_{u}$.

Proof. [1, p. 22].

(Observe that $\mathscr{M}_{\mu}$ is the collection of sets $A \subset E$ such that there are $B_{1} \subset A \subset B_{2}$, $B_{1} B_{2} \in \mathscr{E}$ and $\mu\left(B_{1}\right)=\mu\left(B_{2}\right)$. Necessarily, then, $\tilde{\mu}(A)=\tilde{\mu}\left(B_{l}\right)$, while $\tilde{\mu}(A)=0$ if and only if $A \subset B \in \mathscr{E}$ and $\mu(B)=0$.)

COROllary 1. If $\mathscr{A}$ is a $\sigma$-algebra on $E$ containing $\mathscr{K}(E), \mu$ is a measure on $\mathscr{A}$, and $A$ is capacitable (w.r.t. $\left.\mu^{*}\right)$, then $A \in \mathscr{M}_{\mu}$ and $\tilde{\mu}(A)=\mu^{*}(A)$. Therefore, each $K$-analytic subset of $E$ is in $\mathscr{M}_{\mu}$.

Proof. This follows from Theorems 2, 3, and 4.

The two most familiar such $\mathscr{A}$ are those defined in (b) and (c) of $\S 1$. Here we have this scheme:

$$
(E, \mathscr{F}(E)) \stackrel{i}{\longrightarrow}(E, \mathscr{B}(E)) .
$$

Corollary 2. Suppose that $E$ is $K$-analytic. Then each $\mu$ on $\mathscr{B}(E)$ can be uniquely extended to $\tilde{\mu}$ on $\mathscr{F}(E)$. Moreover, $\mathscr{M}_{\mu}=\mathscr{M}_{\tilde{\mu}}$.

Proof. Since each closed $F \subset E$ is $K$-analytic and each $K$-analytic subset is in $\mathscr{M}_{\mu}$, we have $F \in \mathscr{M}_{\mu}$. Therefore $\mathscr{F}(E) \subset \mathscr{M}_{\mu}$. Extend $\mu$ to $\mathscr{M}_{\mu}$, and let $\tilde{\mu}$ be the restriction to $\mathscr{F}(E)$. Since any set in $\mathscr{F}(E)$ of $\tilde{\mu}$-measure 0 is contained in some set of $\mu$-measure 0 , we have $\mathscr{M}_{\tilde{\mu}} \subset \mathscr{M}_{\mu}$. The reverse inclusion is obvious, since $\tilde{\mu}$ is an extension of $\mu$.

Definition. For a space $E$, let $\mathscr{U}(E)=\bigcap \mathscr{M}_{\mu}$ where the intersection is over all $\mu$ defined on $\mathscr{F}(E)$. These are called the universally measurable sets. 
Note that, for $K$-analytic $E$, we can use $\mathscr{B}(E)$ in place of $\mathscr{F}(E)$. The reason for picking $\mathscr{F}(E)$ is that this choice makes each continuous function a measurable function. Moreover, if $f: E \rightarrow F$ is continuous, then $f:(E, \mathscr{U}(E) \rightarrow(F, \mathscr{U}(F))$ is measurable. This follows from the fact that for each $\mu$ on $\mathscr{F}(E), \tilde{f}(\mu)$ is a measure on $F$ and if $A \in \mathscr{M}_{\tilde{f}(\mu)}$, then $f^{-1}(A) \in \mathscr{M}_{\mu}$.

Definition. Let $M_{+}(E)$ be the set of finite, nonnegative measures on $\mathscr{U}(E)$.

Corollary 2 '. Let $E$ be $K$-analytic and contained in $F$. Then

$$
\mathscr{U}(E)=\{A \cap E: A \in \mathscr{U}(F)\}=\mathscr{U}(F) \mid E .
$$

Proof. First check that $\mathscr{B}(E) \subset \mathscr{U}(F)$, since $E \in \mathscr{U}(F)$ and compact subsets of $E$ are in $\mathscr{U}(F)$. Then let $\mu \in M_{+}(F)$. Now, $\nu=\mu \mid(\mathscr{U}(F) \mid E)$ is defined on $\mathscr{B}(E)$. Suppose that $A \in \mathscr{M}_{v}$. Then there are $B_{1}, B_{2} \in \mathscr{U}(E)$ such that $B_{1} \subset A \subset B_{2}$ and $\nu\left(B_{1}\right)=\nu\left(B_{2}\right)$. Since $B_{1}$ and $B_{2}$ are in $\mathscr{U}(F), A \in \mathscr{M}_{\mu}$. However, since $E \in \mathscr{U}(F)$, any $\nu$ on $\mathscr{U}(E)$ is of the above form, and we have $\mathscr{U}(E) \subset \mathscr{U}(F) \mid E$. The other inclusion is a special case of the result above that each continuous function is measurable.

THEOREM 5. Let $E$ be $K$-analytic and $f$ a continuous function from $E$ to $F$. For $\mu \in M_{+}(E)$ define $\tilde{f}(\mu) \in M_{+}(F)$ by $\tilde{f} \mu(A)=\mu\left(f^{-1}(A)\right)$. Then $f(E) \in \mathscr{U}(F)$ and $\tilde{f} M_{+}(E)=\left\{\mu \in M_{+}(F): \mu\left(f(E)^{\prime}\right)=0\right\}=M_{0}(f(E))$.

Proof. Obviously $f\left(M_{+}(E)\right) \subset M_{0}(f(E))$. Suppose that $\mu \in M_{0}(f(E)) \subset M_{+}(F)$. Then consider the function $I$ on $2^{E}$ defined by $I(A)=\mu^{*} f(A)$. It is easy to see that $I$ is a capacity. Therefore there is an increasing sequence of compact subsets $K_{i} \subset E$ such that

$$
I(E)=\sup I\left(K_{i}\right)=\sup \mu^{*} f\left(K_{i}\right)=\sup \mu f\left(K_{i}\right)=\mu\left(\bigcup f\left(K_{i}\right)\right) .
$$

However $\mu(f(E))=I(E)$. Therefore, $\mu(f(E))=\mu\left(f\left(\cup K_{i}\right)\right)$. It follows that we need verify only that such a $\mu \in M_{0}\left(f\left(\cup K_{i}\right)\right)$ is of the form $f(\sigma)$ for some $\sigma \in M_{+}\left(\bigcup K_{i}\right)$.

Suppose that $\mu \in M_{0}\left(f\left(\cup K_{i}\right)\right)$. Let $\mu_{i}=\mu \mid f\left(K_{i}\right)$. It follows from the HahnBanach theorem and the Riesz theorem that any $\nu_{i} \in M_{+}\left(f\left(K_{i}\right)\right)$ equals $f\left(\sigma_{i}\right)$ for some $\sigma_{i} \in M_{+}\left(K_{i}\right)$. We will also denote by $\sigma_{i}$ the unique extension of $\sigma_{i}$ to $\bigcup K_{n}$ such that the measure of $\bigcup K_{n}-K_{i}$ is zero. Hence we may pick $\sigma_{1}$ such that $\tilde{f}\left(\sigma_{1}\right)=\mu_{1}$ and $\sigma_{i}$ on $K_{i}$ such that $\tilde{f}\left(\sigma_{i}\right)=\mu_{i}-\mu_{i-1}$. (Note that $\mu_{i}-\mu_{i-1}$ is nonnegative.) Let us check that $\sigma_{i+1}\left(f^{-1}\left(f K_{i}\right)\right)=0$. In fact,

$$
\sigma_{i+1}\left(f^{-1}\left(f K_{i}\right)\right)=\tilde{f} \sigma_{i+1} f\left(K_{i}\right)=\nu_{i+1}\left(f\left(K_{i}\right)\right)-\nu_{i}\left(f\left(K_{i}\right)\right)=0 .
$$

Therefore, $\sum \sigma_{i}$ converges to a measure $\sigma$ on $\mathscr{U}\left(\bigcup K_{i}\right)$ such that $f(\sigma)=\mu$.

Corollary 3. Let $E$ be $K$-analytic and $f$ continuous from $E$ onto $F$. Then $f$ maps $M_{+}(E)$ onto $M_{+}(F)$.

4. Topologies on $M_{+}(E)$. For any $E$, we topologize $M_{+}(E)$ by $\mu_{\alpha} \rightarrow \mu$ iff $\int f d \mu_{\alpha} \rightarrow \int f d \mu$ for all bounded continuous real $f$. We have seen that, if $E$ is 
$K$-analytic, and $f$ is continuous from $E$ into $F$, then $f$ maps $M_{+}(E)$ onto $M_{0}(f(E))$. Moreover, $f$ is obviously continuous. This suggests the next theorem.

THEOREM 6. If $E$ is K-analytic and $f$ is a homeomorphism of $E$ into $F$, then $f$ is a homeomorphism of $M_{+}(E)$ onto $M_{0}(f(E))$.

Proof. It follows from Theorem 5 that $f$ is one-one. Hence by the above remark we need only prove $f\left(\mu_{\alpha}\right) \rightarrow f\left(\mu_{0}\right) \Rightarrow \mu_{\alpha} \rightarrow \mu_{0}$.

We first recall the following fact.

Fact. Let $\sigma_{0}$ be in $M_{+}(F)$, and let $K \in \mathscr{K}(F)$ be such that $\sigma_{0}\left(K^{\prime}\right)<\varepsilon$. If $\sigma_{\alpha} \rightarrow \sigma_{0}$ and $U$ is open about $K$, then $\sigma_{\alpha}\left(U^{\prime}\right)<\varepsilon$ for all large $\alpha$. ( $U^{\prime}$ denotes the complement of $U$.)

Proof of fact. Let $\varphi$ be a continuous function on $F$ such that $\varphi\left(U^{\prime}\right)=1, \varphi(K)=0$, and $0 \leqq \varphi \leqq 1$. Then if $\alpha$ is large and $\delta>0$,

$$
\delta+\int \varphi d \mu_{0}>\int \varphi d \sigma_{\alpha} \geqq \int_{U^{\prime}} \varphi d \sigma_{\alpha}=\sigma_{\alpha}\left(U^{\prime}\right)
$$

However, $\int \varphi d \sigma_{0} \leqq \sigma_{0}\left(K^{\prime}\right)$. Therefore, $\sigma_{\alpha}\left(U^{\prime}\right)<\delta+\sigma_{0}\left(K^{\prime}\right)$. If $\delta=\varepsilon-\sigma_{0}\left(K^{\prime}\right)$, then $\sigma_{\alpha}\left(U^{\prime}\right)<\varepsilon$, which completes the proof of the fact.

Now suppose that $\tilde{f}\left(\mu_{\alpha}\right) \rightarrow \tilde{f}\left(\mu_{0}\right)$ in $M_{+}(F)$. Let $g$ be a bounded continuous real function on $E$. Let $\varepsilon>0$, and $K$ a compact subset of $E$ such that $\mu_{0}\left(K^{\prime}\right)<\varepsilon$. There is a function $g_{0}$ on $E$ of the form $g_{0}=\mathrm{h} \circ f$, where $h$ is a bounded continuous function on $F$, such that $\left\|\left(g-g_{0}\right) \mid K\right\|<\varepsilon$. This follows from the Stone-Weierstrass theorem. Let $U$ be an open set about $K$ such that $\left\|\left(g_{0}-g\right) \mid U\right\|<\varepsilon$. Then

$$
\left|\int g d \mu_{\alpha}-\int g d \mu_{0}\right| \leqq\left|\int_{U} g d\left(\mu_{\alpha}-\mu_{0}\right)\right|+2 \varepsilon\|g\|
$$

if $\alpha$ is large, by the fact. Also,

$$
\left|\int_{U} g d\left(\mu_{\alpha}-\mu_{0}\right)\right| \leqq \varepsilon\left(\mu_{\alpha}(U)+\mu_{0}(U)\right)+\left|\int_{U} g_{0} d\left(\mu_{\alpha}-\mu_{0}\right)\right| .
$$

Now, $\left|\int_{U} g_{0} d\left(\mu_{\alpha}-\mu_{0}\right)\right| \leqq\left|\int g_{0} d\left(\mu_{\alpha}-\mu_{0}\right)\right|+\left\|g_{0}\right\| 2 \varepsilon$, if $\alpha$ is large. Also,

$$
\int g_{0} d\left(\mu_{\alpha}-\mu_{0}\right)=\int h d\left(\tilde{f}\left(\mu_{\alpha}\right)-f^{x}\left(\mu_{0}\right)\right)
$$

by Corollary $2^{\prime}$, and

$$
\left|\int h d\left(\tilde{f}\left(\mu_{\alpha}\right)-\tilde{f}\left(\mu_{0}\right)\right)\right| \leqq\|h\| 2 \varepsilon
$$

if $\alpha$ is large. Hence, if a real number $T$ is chosen so that $\mu_{\alpha}(U)+\mu_{0}(U) \leqq \mu_{\alpha}(E)$ $+\mu_{0}(E) \leqq 2 T$ for large $\alpha$, we find that

$$
\left|\int g d \mu_{\alpha}-\int g d \mu_{0}\right| \leqq 2 \varepsilon\left(\|g\|+T+\left\|g_{0}\right\|+\|h\|\right)
$$


for large $\alpha$. This completes the proof, since we may suppose that $\|h\|$ and $\left\|g_{0}\right\|$ are $\leqq\|g\|+\varepsilon$.

REMARK. If a compact Hausdorff $E$ is topologized by a collection of bounded real functions $\mathcal{O}$ of cardinality $\leqq \aleph, \aleph_{0} \leqq \aleph$, then $M_{+}(E)$ has the same property.

Hint. Use polynomials in elements of $\mathcal{O}$ with rational coefficients and the StoneWeierstrass theorem to get a norm dense family in $C(E)$ which topologizes $M_{+}(E)$ and has cardinality $\leqq \aleph$.

THEOREM 7. If a Hausdorff $E$ is $K$-analytic and is topologized by a collection of bounded real functions of cardinality $\leqq \aleph$, then $M_{+}(E)$ is K-analytic and is topologized by a collection of bounded real functions of cardinality $\leqq \mathcal{K} .\left(\boldsymbol{N}_{0} \leqq \boldsymbol{K}\right.$.)

Proof. Let $f$ map $E$ homeomorphically into a compact space $K$ with the property above. (By the usual duality argument used for compactifications, if a Hausdorff $E$ is topologized by such a family, then $E$ is homeomorphic to a subspace of a compact space with the same property.) Then $f$ maps $M_{+}(E)$ homeomorphically onto $M_{0}(f(E)) \subset M_{+}(K)$. Therefore, $M_{+}(E)$ has the property concerning the topologizing functions.

The reader may omit the remainder of this proof, since it is not used for the theorem. It remains to show that $M_{+}(E)$ is $K$-analytic. From Corollary 3, it suffices to prove this for each $E$ which is a $K_{\sigma \delta}$, say $E=\bigcap E_{i}, E_{i}$ is a $K_{\sigma}$. In this case, $M_{+}(E)=\bigcap M_{0}\left(E_{i}\right)$. Since $M_{0}\left(E_{i}\right)$ is homeomorphic to $M_{+}\left(E_{i}\right)$, we need only establish the result for $K_{\sigma}$ 's. Moreover, if $E=\bigcup K$ we may even assume that $K_{i} \cap K_{j}=\varnothing$ if $i \neq j$, since any $K_{\sigma}$ is the continuous image of a countable disjoint union of compact sets and Corollary 3 applies again.

Hence, suppose that $E=\bigcup K_{i}, K_{i}$ compact, and $K_{i} \cap K_{j}=\varnothing$ if $i \neq j$. Let $E^{*}$ be the one point compactification of $E$. Let $\varepsilon>0$, and let

$$
M_{\varepsilon}\left(K_{i}\right)=\left\{\mu \in M_{+}\left(E^{*}\right): \mu\left(K_{i}^{\prime}\right) \leqq \varepsilon\right\} .
$$

Then $M_{\varepsilon}\left(K_{i}\right)$ is closed in $M_{+}\left(E^{*}\right)$ as we will show. In fact, $K_{i}$ is open in $E^{*}$ and there is a continuous real function $f$ with $f\left(K_{i}^{\prime}\right)=1$ and $f\left(K_{i}\right)=0$. If $\mu$ is not in $M_{\varepsilon}\left(K_{i}\right)$ and $2 \alpha=\mu\left(K_{i}^{\prime}\right)-\varepsilon$, then $\left\{\nu \in M_{+}(E): \int f d \nu>\varepsilon+\alpha\right\}$ is an open set about $\mu$ which is disjoint from $M_{\varepsilon}\left(K_{i}\right)$. Therefore $M_{\varepsilon}\left(K_{i}\right)$ is closed in $M_{+}\left(E^{*}\right)$. As $M_{+}\left(E^{*}\right)$ is a $K, M_{\varepsilon}\left(K_{i}\right)$ is a $K$.

Now observe that $\bigcup M_{\varepsilon}\left(K_{i}\right)$ is a $K_{\sigma}$ in $M_{+}\left(E^{*}\right)$ and that $M_{0}\left(\cup K_{i}\right)$ is the intersection of a countable number of these. This shows that $M_{+}(E)$ is a $K_{\sigma \delta}$, since $M_{+}(E)$ is homeomorphic to $M_{0}\left(\cup K_{i}\right)$, and completes the proof.

5. Convex sets. For a convex set $K$, an extreme point is a point that is not interior to any segment in $K$. The main connection between a compact convex set $K$ and the measures on its extreme points is given in the theorem below. For a space $F$, let $P(F)$ denote the probability measures in $M_{+}(F)$. That is, $\mu \in P(F)$ iff $\mu \in M_{+}(F)$ and $\mu(F)=1$. Note that $P(F)$ is closed in $M_{+}(F)$, so that the above 
theorems apply to $P(F)$ as well as $M_{+}(F)$. Also, if $A \in \mathscr{U}(F)$, let $P_{0}(A)$ be the set of $\mu \in P(F)$ such that $\mu\left(A^{\prime}\right)=0$. For a compact convex set $K$ in a locally convex vector space $V$, it is a familiar consequence of the Hahn-Banach theorem that the continuous real linear functions on $V$ topologize $K$. Therefore, the continuous real affine functions $A(K)$ on $K$, defined by the condition $f(t x+(1-t) y)=t f(x)+(1-t) f(y)$ for $0 \leqq t \leqq 1$ and $x, y \in K$, topologize $K$. This is the property of $K$ we use. Since it is equivalent to the hypothesis that $K$ is a compact convex subset of a locally convex vector space, one may use it as the definition.

Now for each $\mu \in P(K)$, it follows easily that there is a point $r \mu \in K$ such that $f(r \mu)=\int f d \mu$ for each $f \in A(K)$. If $K$ is Hausdorff, $r \mu$ is uniquely determined by $\mu$. In that case the map $\mu \rightarrow r \mu$ is continuous.

THEOREM 8. Let $E$ be the set of extreme points of such a Hausdorff compact convex $K$. Let $A$ be a $K$-analytic subset of $K$ which contains $E$. Then for each $x \in K$ there is a $\mu \in P_{0}(A)$ such that $x=r_{\mu}$. That is, $\mu \rightarrow r_{\mu}$ maps $P_{0}(A)$ onto $K$.

Proof. [1, p. 233] where one also will see why such a measure is said to be maximal.

We now state the theorem.

THEOREM. Suppose $K$ and $E$ are as above. If $E$ is the continuous image of a $K$ analytic set $X$ and if $X$ is topologized by a collection of functions of cardinality $\leqq \aleph, \aleph_{0} \leqq \mathbb{N}$, then $K$ is topologized by a collection of functions of cardinality $\leqq \aleph$.

Proof. We show that $P(X) \stackrel{1}{\rightarrow} P(E) \stackrel{2}{\rightarrow} P_{0}(E) \stackrel{3}{\rightarrow} K$ is a sequence of onto maps. In fact, 1 is onto by Corollary 3,2 is onto by Theorem 5 , and 3 is onto by Theorem 8. Therefore, $K$ is the continuous image of $P(X)$ which is a space topologized by a collection of functions of cardinality $\leqq \aleph$, as we see from Theorem 7 . The following observation completes the proof.

Observation. If a compact Hausdorff space $K$ is the continuous image under $f$ of a space $Y$ which is topologized by a collection of functions $\mathcal{O}$ of cardinality $\leqq \mathbb{N}, \boldsymbol{\aleph}_{0} \leqq \mathbb{N}$, then $K$ has the same property.

Proof. Let us consider this property that two subsets $A$ and $B$ of $Y$ may have: There are a finite number of functions in $\mathcal{O}$ and a finite number of closed intervals with rational endpoints such that both $A$ and $B$ are intersections of the inverse images of some of these intervals by some of these functions; and $f(A) \cap f(B)=\varnothing$.

For each such pair $A$ and $B$ pick a continuous function $g_{A B}$ on $K$ such that $0 \leqq g_{A B} \leqq 1$ and $g_{A B}(f(A))=0, g_{A B}(f(B))=1$. There are $\leqq \mathbb{N}$ such $g_{A B}$. It is easy to see that such functions separate points on $K$, since $\mathcal{O}$ topologizes $Y$. Since there are $\leqq \mathbb{N}$ such $g_{A B}$ which separate points and since $K$ is compact, this completes the proof.

CoROLlary. If $E$ and $K$ are as above and $E$ is the continuous image of a complete separable metric space $X$, then $K$ is metrizable. 
Proof. A space is separable metric if and only if it is topologized by a collection of functions of cardinality $\leqq \boldsymbol{N}_{0}$. Consequently, the corollary follows from the theorem as soon as one checks that $X$ is $K$-analytic. This follows from the fact that, under our assumptions, $X$ has a metric compactification and is a $G_{\delta}$ in this compactification; hence, $X$ is a $K_{\sigma \delta}$ in this compactification.

\section{REFERENCES}

1. Paul A. Meyer, Probability and potentials, Blaisdell, Waltham, Mass., 1966. MR 34 \#5119.

2. M. Sion, Topological and measure-theoretic properties of analytic sets, Proc. Amer. Math. Soc. 11 (1960), 769-776. MR 24 \#A1359.

3. - On analytic sets in topological spaces, Trans. Amer. Math. Soc. 96 (1960), 341-354. MR 24 \#A1357.

UNIVERSITY OF WASHINGTON,

Seattle, Washington 98105 